\title{
Beat-induced fluctuations in auditory cortical beta-band activity: using EEG to measure age-related changes
}

\author{
Laura K. Cirelli ${ }^{1}$, Dan Bosnyak ${ }^{1}$, Fiona C. Manning ${ }^{1}$, Christina Spinelli ${ }^{1}$, Céline Marie ${ }^{1}$, Takako Fujioka ${ }^{2,3}$, \\ Ayda Ghahremani ${ }^{1}$ and Laurel J. Trainor ${ }^{1,2,4}$ *
}

\author{
${ }^{1}$ Department of Psychology, Neuroscience \& Behaviour, McMaster University, Hamilton, ON, Canada \\ ${ }^{2}$ McMaster Institute for Music and the Mind, McMaster University, Hamilton, ON, Canada \\ ${ }^{3}$ Centre for Computer Research in Music and Acoustics, Stanford University, Stanford, CA, USA \\ ${ }^{4}$ Rotman Research Institute, Baycrest Hospital, Toronto, ON, Canada
}

\section{Edited by:}

Jessica A. Grahn, University of

Western Ontario, Canada

Reviewed by:

Erin E. Hannon, University of Nevada

Las Vegas, USA

Joel Snyder, University of Nevada Las Vegas, USA

\section{*Correspondence:}

Laurel J. Trainor, Department of

Psychology, Neuroscience \&

Behaviour, McMaster University, 1280

Main Street West, Hamilton,

ON L8S 4K1, Canada

e-mail: ljt@mcmaster.ca
People readily extract regularity in rhythmic auditory patterns, enabling prediction of the onset of the next beat. Recent magnetoencephalography (MEG) research suggests that such prediction is reflected by the entrainment of oscillatory networks in the brain to the tempo of the sequence. In particular, induced beta-band oscillatory activity from auditory cortex decreases after each beat onset and rebounds prior to the onset of the next beat across tempi in a predictive manner. The objective of the present study was to examine the development of such oscillatory activity by comparing electroencephalography (EEG) measures of beta-band fluctuations in 7-year-old children to adults. EEG was recorded while participants listened passively to isochronous tone sequences at three tempi $(390,585$, and $780 \mathrm{~ms}$ for onset-to-onset interval). In adults, induced power in the high beta-band (20$25 \mathrm{~Hz}$ ) decreased after each tone onset and rebounded prior to the onset of the next tone across tempo conditions, consistent with MEG findings. In children, a similar pattern was measured in the two slower tempo conditions, but was weaker in the fastest condition. The results indicate that the beta-band timing network works similarly in children, although there are age-related changes in consistency and the tempo range over which it operates.

Keywords: neural oscillation, time perception, electroencephalography (EEG), musical rhythm, child development

\section{INTRODUCTION}

Humans often spontaneously match the timing of their movements to the timing of a predictable external stimulus, such as that found in most music (Drake et al., 2000b; see Repp and Su, 2013 for a review). Despite the ease with which people entrain to auditory beats, it is a complex process. First, the underlying beat must be extracted from the auditory stimulus, then communicated to the motor system, and finally the motor output is made in a predictive fashion, time-locked to the ongoing beat (see Zatorre et al., 2007; Repp and Su, 2013 for reviews). Rhythm perception and production are thought to share a common timing mechanism (Ivry and Hazeltine, 1995; Ivry, 1996; Hommel et al., 2001; Kello, 2003; Repp et al., 2011; Patel and Iversen, 2014) and there is evidence for a bidirectional relationship between these processes. For example, studies using functional magnetic resonance imaging (fMRI), positron emission tomography (PET) imaging, and magnetoencephalography (MEG) clearly show that listening to predictable temporal patterns recruits both auditory and motor regions of the brain in adults (e.g., Penhune et al., 1998; Schubotz et al., 2000; Schubotz and von Cramon, 2001; Grahn and Brett, 2007; Chen et al., 2008; Bengtsson et al., 2009; Fujioka et al., 2012). Conversely, motor activation influences the perception of auditory information (Phillips-Silver and Trainor, 2007; Su and Pöppel, 2012; Manning and Schutz, 2013). There is even evidence that the way in which infants are bounced to a rhythmic pattern affects their auditory interpretation of that pattern (Phillips-Silver and Trainor, 2005). At the same time, the ability to intentionally entrain movements to an auditory beat in tapping tasks or tasks involving other body movements takes time to mature (e.g., Drake et al., 2000a; Eerola et al., 2006). This raises the question of whether this extended immaturity of rhythmic entrainment reflects an immature ability to perceive regular beats in auditory cortex, or whether it is due to motoric immaturity. In the present study, we begin to address this question by examining whether auditory cortical responses to regular beats are mature in 7-year-old children. Specifically, we use electroencephalography (EEG) to examine auditory cortical entrainment to isochronous sequences at different tempi, bypassing the need for children to make an overt motor response.

The brain encodes different aspects of timing information, processing both interval (duration) and relative (beat-based) timing (e.g., Koch et al., 2009; Grube et al., 2010; Teki et al., 2011). Although young infants process both interval and relative timing, as discussed below, even the most basic aspects of time perception continue to improve for many years. For example, with respect to interval timing, young infants can detect small gaps in auditory stimuli (Werner et al., 1992; Trehub et al., 1995; Trainor et al., 2001; Smith et al., 2006) and detect duration differences in speech (e.g., Eilers et al., 1984), but thresholds do not reach adult levels until at least into the school age years, particularly when timing is compared across stimuli with different frequencies (Trehub et al., 1995).

There is evidence that even young infants are sensitive to relative (beat-based) timing when there is no requirement for motor 
entrainment. For example, they recognize rhythms across changes in tempo and frequency range (Chang and Trehub, 1977; Demany et al., 1977; Trehub and Thorpe, 1989) and extract the underlying regular beat (Hannon and Trehub, 2005a,b; Winkler et al., 2009). Older children can detect changes in underlying regularities in rhythmic stimuli (e.g., Baruch et al., 2004) and determine whether an isochronous beat train superimposed on a musical excerpt is at the correct tempo and phase (Einarson and Trainor, 2013). However, the ability to entrain movements to rhythm patterns or regular predictable beats remains immature for a long time. Infants as young as 5 months of age will move their bodies rhythmically and with more vigor when listening to music or isochronous tones as opposed to non-musical speech, however, these movements are not synchronous with the beat of the music (Zentner and Eerola, 2010). Two- and four-year-old children spontaneously sway and hop to musical stimuli, but do not adjust the tempo of this movement in response to fluctuations in stimulus timing (Eerola et al., 2006). Synchronization abilities do improve throughout childhood, but even 10-year-olds do not tap to an auditory rhythm as consistently as do adults (Drake et al., 2000a). In addition, even as these synchronization abilities improve, children's spontaneous tapping rates remain faster than those of adults (Drake et al., 2000a; Baruch etal., 2004; McAuley et al., 2006). Together these studies suggest that although young children are inclined to move in a rhythmic fashion in the presence of a predictable auditory beat, they are unable to consistently entrain their movements to the underlying pulse. It is unclear if this is because auditory cortical entrainment mechanisms are mature in children and the immaturity lies in connections between auditory and motor areas, or whether the perception of entrainment in auditory cortex itself has a protracted developmental trajectory. The present study begins to address this question by focusing on the maturity of auditory cortical activity during rhythm perception in children.

One popular theoretical framework for understanding auditory rhythm perception is Dynamic Attending Theory (Jones and Boltz, 1989; Large and Jones, 1999). This theory proposes that internal oscillators entrain to external auditory rhythms in a way that focuses attentional resources at important points in time. For example, in an isochronous sequence, attention is focused toward the predicted onset time of the next tone. Neurophysiological evidence suggests that the underlying mechanism for predictive timing is reflected in oscillatory neural activity (Snyder and Large, 2005; Lakatos et al., 2008; Fujioka et al., 2009, 2012; Iversen et al., 2009; Arnal, 2012; Arnal and Giraud, 2012; Nozaradan et al., 2012). Neural oscillations involved in predictive timing appear to span the delta $(0.5-3 \mathrm{~Hz})$, beta $(15-30 \mathrm{~Hz})$, and gamma $(30-100 \mathrm{~Hz})$ ranges. Human beat processing is optimal when the tempo of the stimulus is in the range of $1-3 \mathrm{~Hz}$ (e.g., Fraisse, 1982; Drake and Botte, 1993; Large, 2008) and stimuli consisting of rhythmic patterns give rise to oscillations in the EEG in this delta range most likely because it directly reflects the frequency of the beat or its metric organization as phase-locked neural activities (Nozaradan et al., 2012). These oscillations reflect to some extent stimulus event onsets, but instructions to imagine different metric structures (such as accents every second beat vs. every third beat) while listening to the same stimulus modifies oscillation amplitudes, indicating that the oscillations can also reflect internally generated timing expectations (Nozaradan et al., 2011). Such modulation of oscillatory neural activities also contributes to the phase-locked neural activities across a wide range of brain areas related to auditory and motor systems (Fujioka et al., 2010).

Oscillations in the beta-band appear to be particularly important for auditory rhythmic entrainment (Fujioka et al., 2009, 2012; Iversen et al., 2009; Patel and Iversen, 2014), which is interesting given that the beta-band is also implicated in regulating the initiation of movement (e.g., Hari and Salmelin, 1997; Pfurtscheller and Lopes da Silva, 1999; Gilbertson et al., 2005). Furthermore, there is coupling between different oscillation frequencies, such that the amplitude of beta oscillations can vary according to the phase of a delta rhythm (Lakatos et al., 2008; Fujioka et al., 2012). While delta oscillations tend to phase lock to onsets of predictable sound events, beta oscillations typically do not. Thus, averaging evoked MEG or EEG responses to the sound events in a presented rhythm pattern may remove most activation in the beta-band. However, if single-trial analysis is used, fluctuations in betaband power are evident (induced beta oscillations) that follow the beat of the rhythm pattern. Fujioka et al. (2009, 2012) measured induced beta responses to isochronous sound streams presented at three stimulus rates (tempi). They found beta desynchronization after each tone followed by a rebound whose timing depended on stimulus rate, suggesting that the rebound rate was predictive of the expected onset time of the next tone. The authors interpreted these results as being consistent with literature suggesting that beta desynchronization reflects a change in event processing (Pfurtscheller and Lopes da Silva, 1999), while synchronized rebound is associated with a "reset of an existing cortical network" (Pfurtscheller et al., 2005). Furthermore, this modulation of induced beta activity was found not only in auditory cortex, but also in a number of cortical and subcortical areas of the motor network. As participants were instructed not to move throughout the experiment, this suggests that even the perception of auditory rhythms may involve processing loops between auditory and motor regions.

Induced oscillatory neural activity representing beat perception has not previously been measured in children. In the present study we compared induced beta oscillatory activity from auditory cortex in 7.5-year-old children and adults while they passively listened to isochronous tones at three tempi. We expected that induced beta oscillations would be qualitatively similar across the age groups, but that differences might exist in the extreme (fastest or slowest) tempo conditions. The present study used EEG to examine betaband activity, as opposed to MEG as in previous studies (Fujioka et al., 2009, 2012). Although spatial resolution is better in MEG compared to EEG, it is much easier to use EEG with children, as the demands for remaining completely still are less critical (Trainor, 2012). Importantly, the use of EEG will allow direct comparison to future studies in younger children and infants.

\section{MATERIALS AND METHODS PARTICIPANTS}

Seventeen normal hearing adults (three males; mean age $=21.1$ years \pm 3.1 ; one left handed) and 16 normal hearing children (eight 
males; mean age $=7.6$ years \pm 0.2 ; one left handed, one ambidextrous) participated in the experiment. Two additional adults and one child were tested, but were excluded due to equipment failure. One additional adult was tested but was excluded due to reported hearing loss.

After the nature of the study was described, each participant (and for the children, also the parent) provided informed consent and completed a short survey outlining the participant's hearing and musical history. None of the participants reported any family history of hearing impairment. Musical training was reported for eight of the children $(M=2.1 \pm 1.9$ years of formal instruction for the eight children $)$ and all of the adult subjects $(M=8.9 \pm 4.3$ years of formal instruction).

The children were recruited through the Developmental Studies Database at McMaster University. The procedures used in this study were approved by the McMaster Research Ethics Board.

\section{EEG RECORDINGS}

Each participant was seated comfortably in a sound-attenuating room. 128 electrodes were applied in a cap and their locations digitized (Polhemus Fastrak, Colchester, VT, USA). EEG was continuously recorded at $2048 \mathrm{~Hz}$ filtered DC to $200 \mathrm{~Hz}$ using a Biosemi ActiveTwo amplifier (Cortech Solutions, Wilmington, NC, USA) with an active reference system. Before recording, electrode offset voltage was verified to ensure adequately low electrode impedance.

\section{PROCEDURE}

The tone used for the stimulus sequence was identical to that used by Fujioka et al. (2012), and consisted of a $264 \mathrm{~Hz}$ pure tones with $8 \mathrm{~ms}$ rise and fall times and $45 \mathrm{~ms}$ steady-state duration. The tones were presented in isochronous sequences at the three tempi used by Fujioka et al. (2012), with onset-to-onset intervals of 390, 585, and $780 \mathrm{~ms}$. Each participant experienced all three tempo conditions in each of the two blocks of the experiment, with the order of the tempo condition presentation for each block randomized across participants. In each block, $\sim 350$ tones were presented per tempo, for a total of $\sim 700$ tones per tempo condition across both blocks. These tones were sequenced using the program Presentation (Neurobehavioral Systems, Berkeley, CA, USA), and presented binaurally to participants through ear inserts (Etymotic ER2) at an intensity of $\sim 60 \mathrm{~dB}$ SPL. Participants passively listened to the stimuli while watching a silent movie with subtitles and were asked to minimize movements and eye blinks during testing phases. Once EEG recording began, the entire experiment lasted for about $20 \mathrm{~min}$.

\section{DATA ANALYSIS AUDITORY SOURCE LOCALIZATION}

Using brain electrical source analysis (BESA, Gräfelfing, Germany) software, for each person, the averaged event-related potential (ERP) to the tones in the $780 \mathrm{~ms}$ condition was calculated (the slowest tempo gives the best signal-to-noise ratio in the evoked response; e.g., Näätänen and Picton, 1987). To do this, epochs were extracted from $100 \mathrm{~ms}$ before tone onset to $250 \mathrm{~ms}$ after tone onset, and epochs with high amplitude voltage were rejected such that $80 \%$ remained (e.g., see Gander et al., 2010 for use of percentage rejection). The epochs remaining after artifact rejection were averaged, and the resulting evoked response was filtered 1$20 \mathrm{~Hz}$. The baseline was corrected using a $100 \mathrm{~ms}$ window prior to the stimulus onset. In order to examine beta activity from auditory cortex, the first step is to locate this region. For this, the $\mathrm{N} 1$ response was used, as it is a robust response known to be generated primarily in auditory cortex. The N1 response was identified as the most negative peak between 90 and $120 \mathrm{~ms}$ from tone onset in the global field power. Two symmetrical regional sources were fit, in each individual, to a window $\pm 10 \mathrm{~ms}$ around the identified peak and then converted into independently oriented single sources. The right source position and orientation was used as a spatial filter in the subsequent analyses. All participants' final source locations were consistent with expected locations in the auditory cortex (see Figure $\mathbf{1}$ for averaged dipole locations).

\section{INDUCED RESPONSE}

Raw data were filtered using each subject's right auditory cortex source as a spatial filter, and further analyses were performed on this source waveform. These data were epoched around each tone according to tempo condition (390 condition: -200 to $600 \mathrm{~ms} ; 585$ condition: -300 to $900 \mathrm{~ms} ; 780$ condition: -400 to $1200 \mathrm{~ms}$ ). All
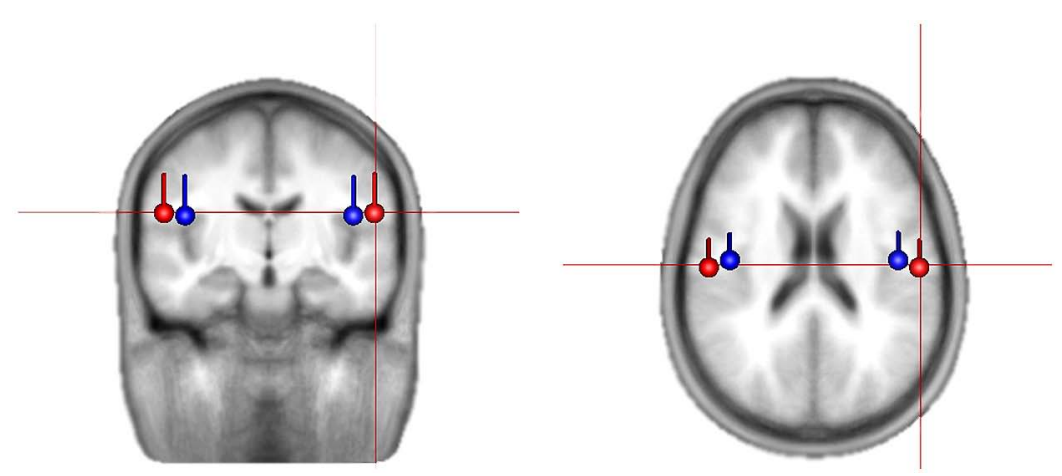

FIGURE 1 | Average location and orientation of the equivalent current dipole sources fitted in the bilateral auditory cortical areas for children (in red) and adults (in blue) that are used as spatial filter in extracting source activities. 
individual epochs were down-sampled at $1000 \mathrm{~Hz}$, filtered from 3 to $200 \mathrm{~Hz}$, and exported for further processing in MATLAB.

Artifact rejection was performed in MATLAB by eliminating epochs with high amplitude voltage such that $80 \%$ of the epochs remained in each condition, and further analyses were done on these epochs. For each tempo condition, the waveforms remaining after artifact rejection were averaged to obtain an estimate of the evoked response, and this was subtracted from each individual epoch to obtain an estimate of the non-phase-locked induced response on each trial.

The individual trials, representing induced activity, were then analyzed in the frequency domain using FFTs on 200 logarithmically spaced frequencies between 2 and $50 \mathrm{~Hz}$ with a sliding Gaussian window ranging between 2 and 4 carrier frequency periods in length. These power spectra were averaged, and for each frequency, the calculated power was normalized to the average power across the beat window (tone onset to subsequent tone onset) and then displayed as a percent change in power over time. The spectrograms for each group (children, adults) and tempo condition were reduced to display one tone onset to the next, from 4 to $40 \mathrm{~Hz}$ (see Figure 2). For analysis, power changes in the bands 15-20 Hz (low beta) and 20-25 Hz (high beta) were averaged over frequency for each individual participant. The group averages for children and adults for each tempo condition are displayed in Figures 3 and $\mathbf{4}$ (high beta and low beta).

\section{RESULTS}

Figure 3 shows fluctuations in induced power for adults and children for each tempo condition for high beta $(20-25 \mathrm{~Hz})$ and Figure 4 for low beta $(15-20 \mathrm{~Hz})$. Adults showed a clear event-related desynchronization after tone onset in induced beta power for all three tempo conditions, followed by a rebound prior to the onset of the next tone (see Figure 2), similar to the previous findings reported using MEG (Fujioka et al., 2009, 2012). As with the previous MEG results, the presence of these patterns of desynchronization and rebound suggests that beta-band oscillations coming from auditory cortex reflect prediction of the timing of upcoming beats. Similarly, patterns of beta desynchronization and rebound can be seen also in the child data for the two slower tempo conditions (585, $780 \mathrm{~ms}$ ), but there is little evidence of this pattern at the fastest tempo (390 ms). In general, the pattern of desynchronization followed by rebound in Figures 3 and $\mathbf{4}$ appears to occur most strongly around $20-25 \mathrm{~Hz}$ in adults but around $15-20 \mathrm{~Hz}$ in children.

Finally, in adults, the tempo-dependent beta fluctuations are clearer for high than for low beta, especially for the fastest tempo (390 ms). One-sample $t$ tests were used to find the time intervals during which the power change differed significantly from zero in each of the three tempo conditions. With the adult data, these $t$ tests confirmed a significant dip in beta synchronization following tone onset for all three tempi in high beta and for the two slower
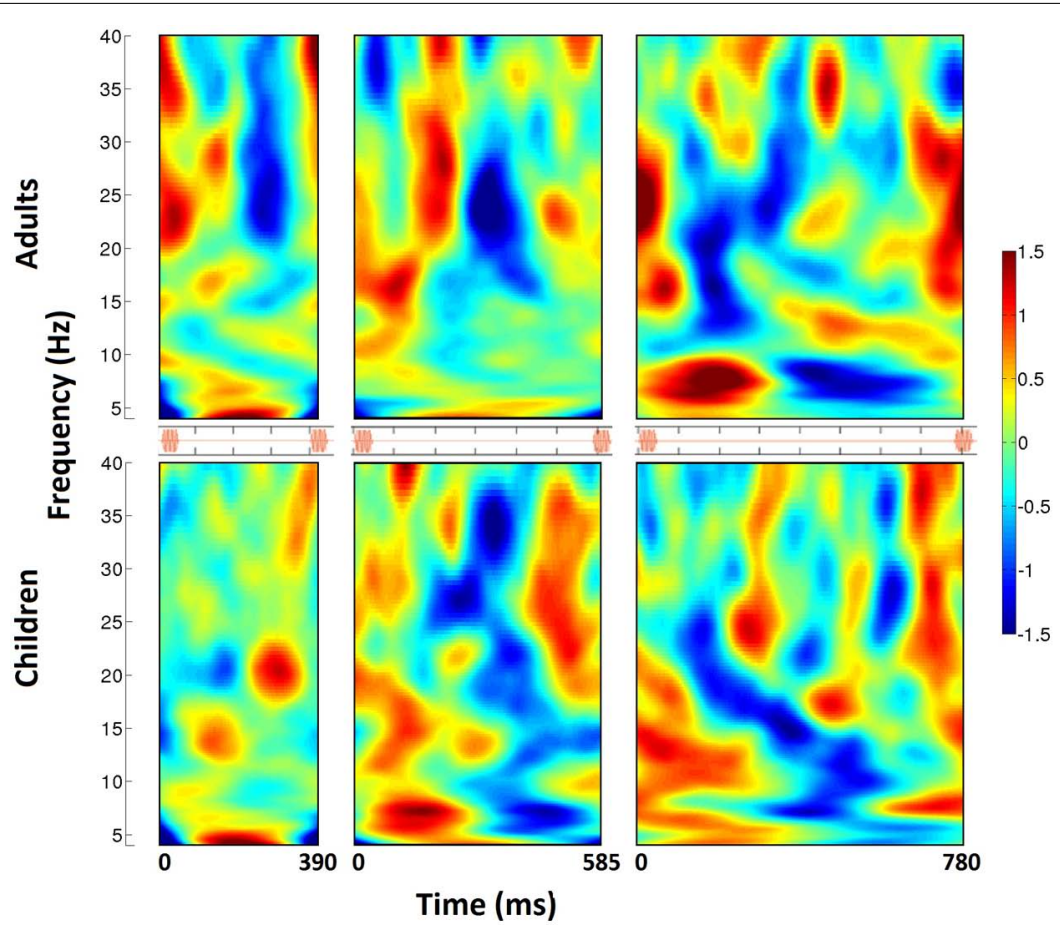

FIGURE 2 | The time-frequency spectrogram of percent power changes from right auditory cortex spatial filter for three tempo conditions $(390$, $\mathbf{5 8 5}, \mathbf{7 8 0} \mathbf{~ m s}$ from left to right). Adults are shown on the top, and children on the bottom. Analyses span from tone onset to subsequent tone onset, as visualized in the orange stimulus onset indicators for each tempo condition.
From the spectrograms, a pattern of induced beta-band desychronization (negative percent change values) following each tone and a rebound (positive percent change values) before the onset of the next beat can be visualized. The timing of this pattern varies across tempo, and is stronger and more consistent in children compared to adults. 

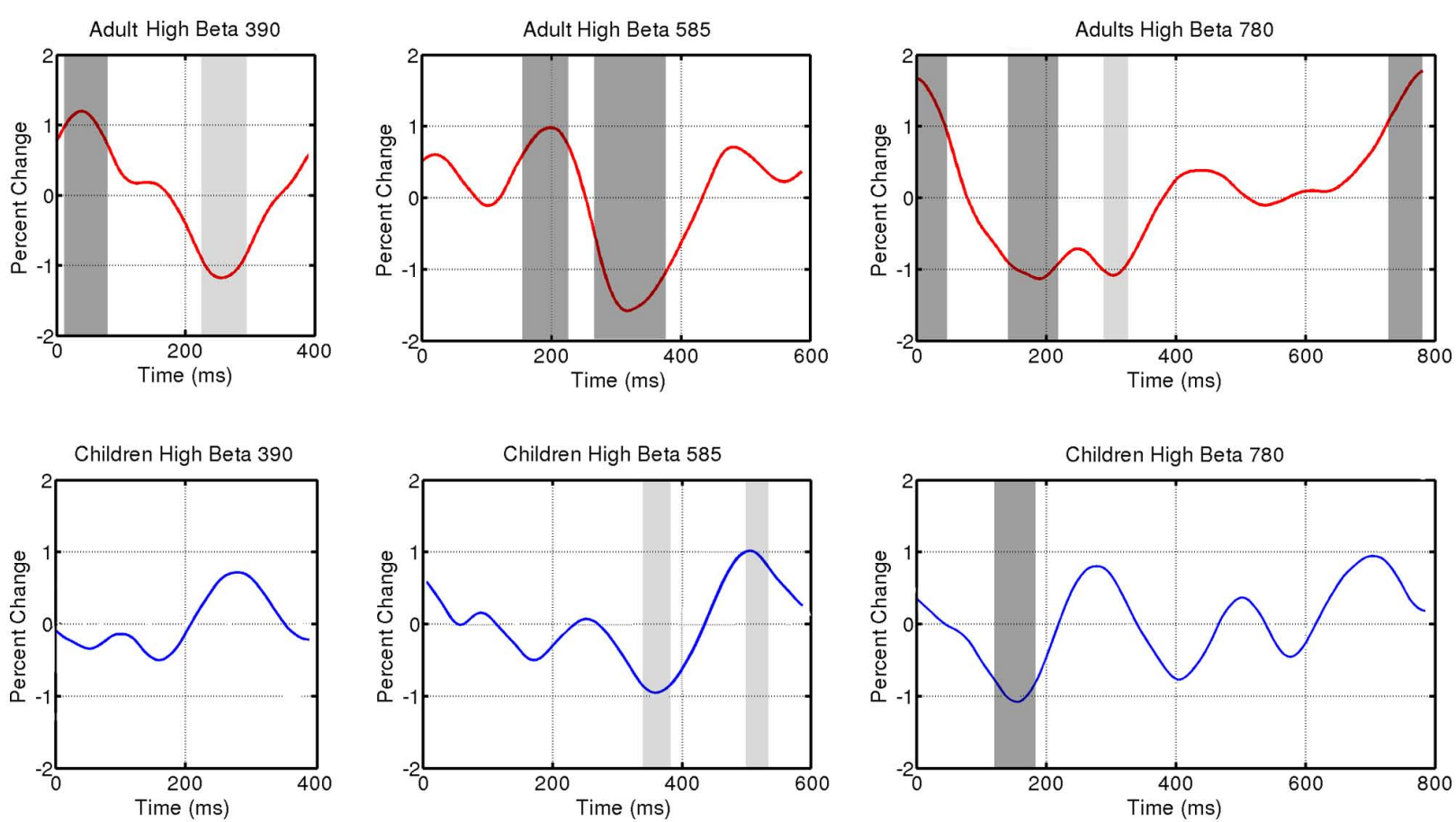

FIGURE 3 | Induced power fluctuation in high beta (20-25 Hz) activity for the three tempo conditions (390, 585, 780 ms) and two groups (adults and children). Running one-sample $t$ tests were used to determine when group fluctuations differed from zero. Light gray represents $p<0.05$. Dark gray represents $p<0.01$.

tempi in low beta (see Figures $\mathbf{3}$ and $\mathbf{4}$ for time ranges and significance levels). In children, one-sample $t$ tests revealed a significant dip in beta synchronization following tone onset for both high and low beta for the two slower tempi $(585,780 \mathrm{~ms})$, but no significant dip in beta synchronization for the fastest tempo $(390 \mathrm{~ms})$ for either high or low beta.

To further investigate the timing of the beta desynchronization and rebound, for each participant the time of maximum beta desynchronization (i.e., time of minimum beta power) was determined separately for high and low beta for each tempo condition as examined in Fujioka et al. (2012). Half power was defined as half of the difference in power between the maximum synchronization and maximum desynchronization. The time courses of desynchronization and rebound were examined by identifying the time at which half power was reached prior to as well as following the point of maximum desynchronization. Figure 5 shows the timing of these three points (midpoint of desynchronization; point of maximum desynchronization; midpoint of rebound). These three time points served as the dependent variable for three separate ANOVAs, each with tempo (390, 585, and $780 \mathrm{~ms}$ ), group (children and adults) and frequency range (high beta, low beta) as independent variables. For each ANOVA there were no significant interactions and no significant main effects of group or frequency range of the beta-band. There were, however, main effects of tempo on the latency of the midpoint of desynchronization $\left(F_{(2,62)}=8.25, p<0.001\right)$, maximum desynchronization $\left(F_{(2,62)}=18.37, p<0.001\right)$ and midpoint of rebound
$\left(F_{(2,62)}=21.32, p<0.001\right)$. Post hoc analyses revealed that these time points occurred sooner for the $390 \mathrm{~ms}$ condition as compared to the 585 and $780 \mathrm{~ms}$ conditions. This result suggests that the timing of induced beta-band desynchronization and rebound does depend on the tempo, suggesting that these fluctuations are predictive in nature.

\section{DISCUSSION}

The purpose of the present experiment was to compare oscillatory responses from auditory cortex in children and adults to isochronous beat trains with different tempi using EEG. The results reveal a pattern of induced beta-band desynchronization following each tone and a rebound before the onset of the next beat. The timing of these patterns of desynchronization and rebound varied across tempo condition. Thus induced beta-band oscillations showed predictive timing that was generally comparable to previous MEG findings (Fujioka et al., 2012). Furthermore, fluctuations in beta power appeared stronger and more consistent in adults compared to children, especially at the fastest tempo.

There were some differences between the present EEG results and previous MEG results in the details of the timing of the desynchronies. Fujioka et al. (2012), using MEG, found that the latencies of the midpoint of desynchronization and maximum desynchronization occured at consistent timepoints ( $\sim 100$ and $200 \mathrm{~ms}$ after tone onset, respectivly) across tempo conditions, and that only the latency of the midpoint of rebound varied as a function of tempo. In the current EEG study, the latencies of all three of these 

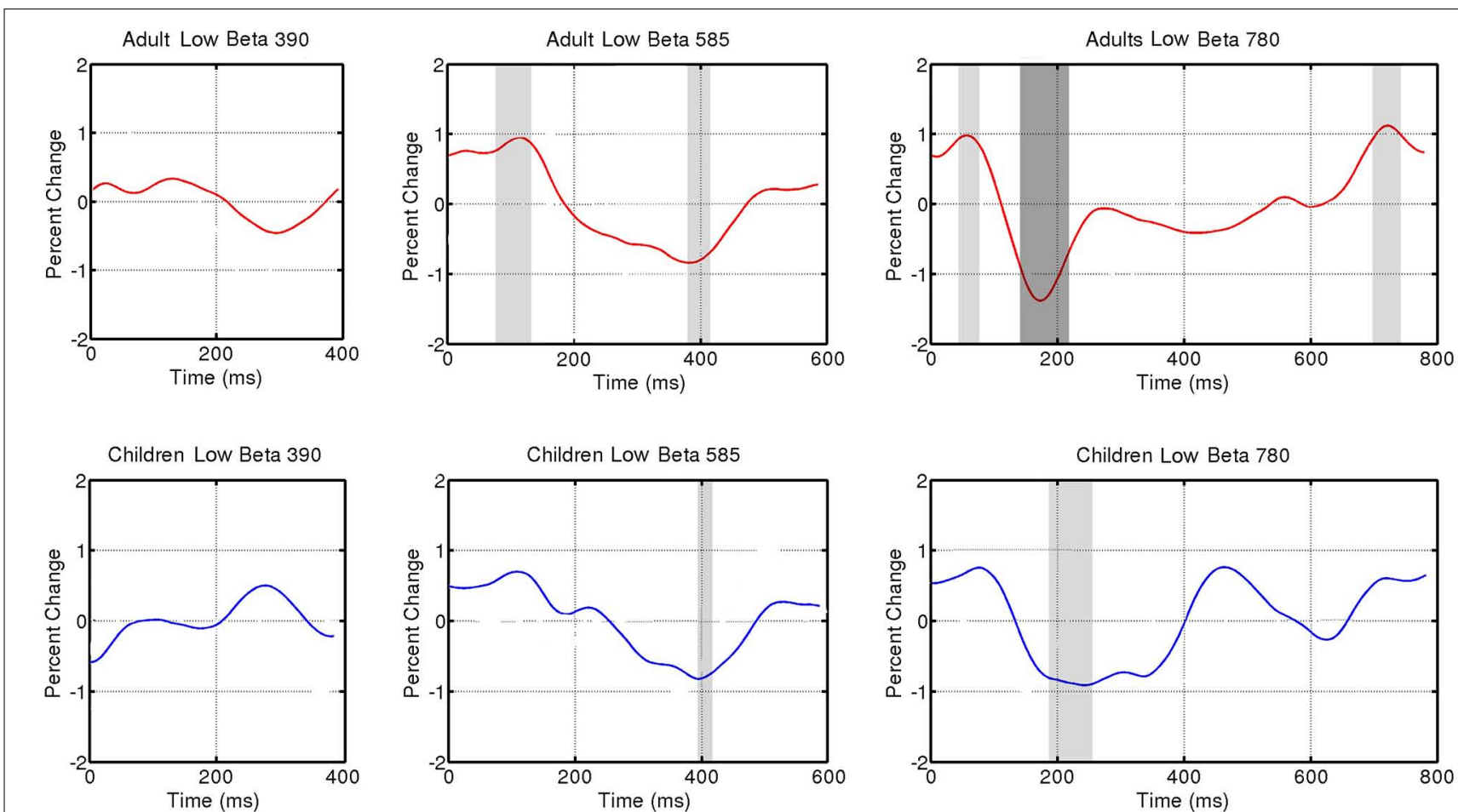

FIGURE 4 | Induced power fluctuation in low beta $(15-20 \mathrm{~Hz})$ activity across the three tempo conditions $(390,585,780 \mathrm{~ms})$ and two groups (adults and children). Running one-sample $t$ tests were used to determine when group fluctuations differed from zero. Light gray represents $p<0.05$. Dark gray represents $p<0.01$.

timepoints varied as a function of tempo. The reasons for these differences are not clear, but might relate to differences between the ability of source filters to resolve spatially separable components in EEG vs. MEG. It is also the case that radial sources visible to EEG are invisible to MEG (Hämäläinen et al., 1993), which can lead to slight differences between the auditory responses recorded by EEG and MEG (Shahin et al., 2007). The MEG study conducted by Fujioka et al. (2012) reported patterns of tempo-dependent beta desynchronization and rebound across many brain regions, prominently including motor areas. The authors interpreted this as automatic activation of motor networks in the analysis of auditory rhythmic information. Despite the fact that we conducted our EEG analyses using source space projection to a region in auditory cortex, the EEG data still may have contained some activity generated in motor regions as well as auditory regions. Auditory and motor regions may have somewhat different time courses of

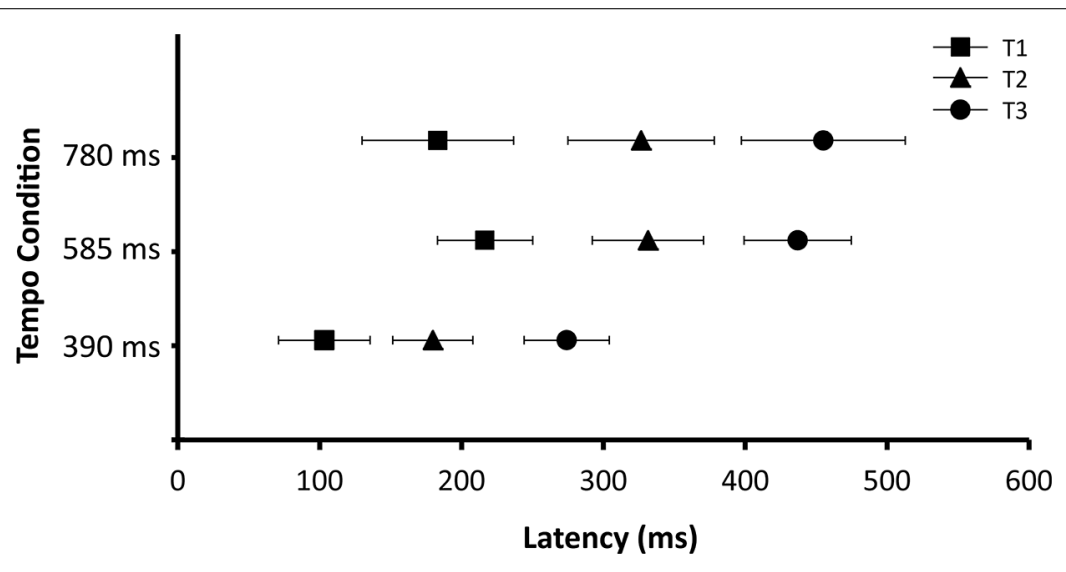

FIGURE 5 | Latency of T1 (midpoint of desynchronization), T2 (maximum point of desynchronization), and T3 (midpoint of rebound). Data are collapsed across age group (adults and children) and beta-band (high and low). Error bars represent $95 \%$ confidence intervals. 
beta desychrony and rebound, which may have been reflected in our data. In any case, the important result is that the pattern of fluctuation in induced beta power that follows the tempo of an isochronous auditory sequence is seen in both the present EEG results and previous MEG results.

In general, the children showed induced beta-band responses that resembled those of the adults. For example, there were no significant differences between children and adults in the latencies of the midpoint of desynchronization, maximum desynchronization, or midpoint of rebound. Across both groups there was a significant effect of tempo, suggesting that the induced beta fluctuations in both adults and children shift in phase depending on the phase of the predictive auditory events. However, the $t$ tests on the amplitudes of the beta power fluctuations revealed differences in the strength and consistency of children's and adult's responses. In high beta, adults showed a significant desynchronization from baseline for all tempo conditions, whereas children only showed significant desynchronization for the two slower tempi. This is interesting given the fact that the range of tempi at which 8-year-old children comfortably tap (both in spontaneously tapping and in tapping entrained to an auditory beat) is not as wide as it is for adults (Drake et al., 2000a). The ability to attend to and reproduce rhythms that occur at tempi outside an ideal tempo (roughly $600 \mathrm{~ms}$ ) develops with age (Drake et al., 2000a).

Children showed less prominent beta desynchronizations compared to adults in the auditory cortex. Also the high-beta modulation was weaker in children than in adults. As these differences seem similar to those observed in the sensorimotor cortices associated with movement tasks between children and adults (Gaetz et al., 2010), our result might indicate a general developmental change in the beta-band oscillatory network. One possible reason for less prominent desychronizations in the group average of children compared to adults is that the time course of beta desynchronization might be more variable in children. We examined this possibility descriptively using circular plots. These plots (see Figure 6) show individual desynchronization data for children and adults across the three tempo conditions (390, 585, or $780 \mathrm{~ms}$ ). Each vector represents one participant, one beat window corresponds to $360^{\circ}$, and the length of the vector represents the amplitude of the percent change in beta power. The direction of the vector represents the phase of the beta power change that repeats once per beat. For adults, there is some variability in phase, but most of the vectors cluster in a similar direction, indicating a similar time course of beta-band power fluctuation, reflected by the length of the average vector (in red). For children, on the other hand, there is considerable variability in phase, so that the average vector is very small. These plots suggest that neural synchronization responses to auditory rhythmic input are considerably more variable in children than in adults.

The results of the present study show that induced beta-band oscillatory responses to rhythmic sound input can be measured in children using EEG, but that such responses from auditory cortex are far from mature at 7 years of age, especially for fast tempi. Thus, the findings suggest that children's immaturity in rhythm production discussed above might be the result of a protracted developmental trajectory for rhythm perception as well as for motoric abilities. Indeed, as children get older, their finger tapping speed becomes faster, in parallel to their motor cortical

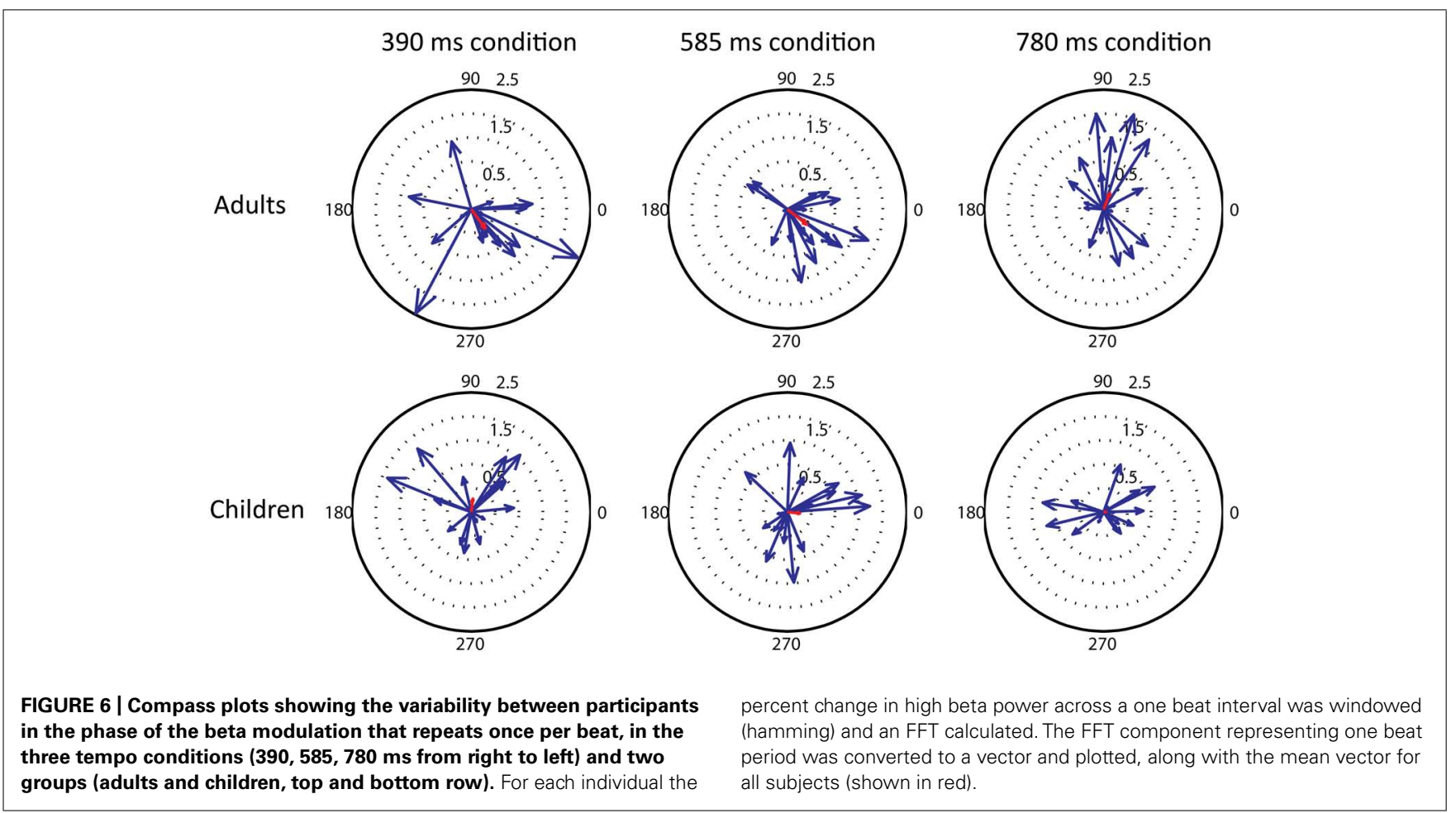


excitability assessed by TMS (Garvey et al., 2003). Especially given the variability in children's responses, an important additional question for future research is the impact of musical training on the maturity and consistency of beta oscillations related to rhythmic processing. Finally, given that EEG has lower requirements for minimizing participant movement during recording, the finding that oscillatory responses can be measured with EEG opens the door for future studies to investigate rhythmic encoding in younger children and infants.

\section{CONCLUSION}

This study is the first to measure induced oscillatory responses to isochronous tone sequences in children. By refining such EEG methods, it is now possible to investigate the developmental trajectory of these oscillatory responses from infancy into adulthood. Children responded in an adult-like manner at slower but not at faster tempi. This finding is consistent with the literature on spontaneous tapping rates and how the tempo range of beat perception increases with age. Future studies can focus on further disentangling the contributions of auditory perception and motoric maturity to beat perception and production.

\section{ACKNOWLEDGMENTS}

This research was funded by a grant from the Canadian Institute of Health Research to Laurel J. Trainor, and by the Social Sciences and Humanities Research Council to Laura K. Cirelli. Céline Marie and Christina Spinelli were supported by postdoctoral and undergraduate award, respectively, from a Natural Sciences and Engineering Research Council of Canada CREATE grant in Auditory Cognitive Neuroscience.

\section{REFERENCES}

Arnal, L. H. (2012). Predicting “when” using the motor system's beta-band oscillations. Front. Hum. Neurosci. 6:225. doi: 10.3389/fnhum.2012.00225

Arnal, L. H., and Giraud, A. L. (2012). Cortical oscillations and sensory predictions. Trends Cogn. Sci. 16, 390-398. doi: 10.1016/j.tics.2012.05.003

Baruch, C., Panissal-Vieu, N., and Drake, C. (2004). Preferred perceptual tempo for sound sequences: comparison of adults, children and infants. Percept. Mot. Skills 98, 325-339. doi: 10.2466/pms.98.1.325-339

Bengtsson, S. L., Ullén, F., Ehrsson, H. H., Hashimoto, T., Kito, T., Naito, E., et al. (2009). Listening to rhythms activates motor and premotor cortices. Cortex 45 , 62-71. doi: 10.1016/j.cortex.2008.07.002

Chang, H., and Trehub, S. E. (1977). Infants' perception of temporal grouping in auditory patterns. Child Dev. 48, 1666-1670. doi: 10.2307/1128532

Chen, J. L., Penhune, V. B., and Zatorre, R. J. (2008). Listening to musical rhythms recruits motor regions of the brain. Cereb. Cortex 18, 2844-2854. doi: 10.1093/cercor/bhn042

Demany, L., McKenzie, B., and Vurpillot, E. (1977). Rhythm perception in early infancy. Nature 266, 718-719. doi: 10.1038/266718a0

Drake, C., and Botte, M. C. (1993). Tempo sensitivity in auditory sequences: evidence for a multiple-look model. Percept. Psychophys. 54, 277-286. doi: 10.3758/BF03205262

Drake, C., Jones, M. R., and Baruch, C. (2000a). The development of rhythmic attending in auditory sequences: attunement, referent period, focal attending. Cognition 77, 251-288. doi: 10.1016/S0010-0277(00)00106-2

Drake, C., Penel, A., and Bigand, E. (2000b). Tapping in time with mechanically and expressively performed music. Music Percept. 18, 1-23. doi: 10.2307/40285899

Eerola, T., Luck, G., and Toiviainen, P. (2006). “An investigation of preschoolers' corporeal synchronization with music," in Proceedings of the 9th International Conference on Music Perception and Cognition, eds M. Baroni, A. R. Addessi, R. Caterina, and M. Costa (Bologna: ICMPC-ESCOM), 472-476.
Eilers, R., Bull, D., Oller, D., and Lewis, D. (1984). The discrimination of vowel duration by infants. J. Acoust. Soc. Am. 75, 1213-1218. doi: 10.1121/1.390773

Einarson, K. M., and Trainor, L. J. (2013). "Five-year-old children's beat perception and beat synchronization abilities," in Frontiers in Human Neuroscience Conference Abstract: 14th Rhythm Production and Perception Workshop, Birmingham 11th13th September 2013. doi: 10.3389/conf.fnhum.2013.214.00017

Fraisse, P. (1982). "Rhythm and tempo," in The Psychology of Music, 1st Edn, ed. D. Deutsch (New York, NY: Academic Press), 149-180. doi: 10.1016/B978-0-12213562-0.50010-3

Fujioka, T., Trainor, L. J., Large, E. W., and Ross, B. (2009). Beta and gamma rhythms in human auditory cortex during musical beat processing. Ann. N. Y. Acad. Sci. 1169, 89-92. doi: 10.1111/j.1749-6632.2009.04779.x

Fujioka, T., Trainor, L. J., Large, E. W., and Ross, B. (2012). Internalized timing of isochronous sounds is represented in neuromagnetic $\beta$ oscillations. J. Neurosci. 32, 1791-1802. doi: 10.1523/JNEUROSCI.4107-11.2012

Fujioka, T., Zendel, B., and Ross, B. (2010). Endogenous neuromagnetic activity for mental hierarchy of timing. J. Neurosci. 30, 3458-3466. doi: 10.1523/JNEUROSCI.3086-09.2010

Gaetz, W., MacDonald, M., Cheyne, D., and Snead, O. C. (2010). Neuromagnetic imaging of movement-related cortical oscillations in children and adults: age predicts post-movement beta rebound. Neuroimage 51, 792-807. doi: 10.1016/j.neuroimage.2010.01.077

Gander, P. E., Bosnyak, D. J., and Roberts, L. E. (2010). Acoustic experience but not attention modifies neural population phase expressed in human primary auditory cortex. Hear. Res. 269, 81-94. doi: 10.1016/j.heares.2010.07.001

Garvey, M. A., Ziemann, U., Bartko, J. J., Denckla, M. B., Barker, C. A., and Wassermann, E. M. (2003). Cortical correlates of neuromotor development in healthy children. Clin. Neurophysiol. 114, 1662-1670. doi: 10.1016/S13882457(03)00130-5

Gilbertson, T., Lalo, E., Doyle, L., Di Lazzaro, V., Cioni, B., and Brown, P. (2005). Existing motor state is favored at the expense of new movement during 13$35 \mathrm{~Hz}$ oscillatory synchrony in the human corticospinal system. J. Neurosci. 25, 7771-7779. doi: 10.1523/JNEUROSCI.1762-05.2005

Grahn, J. A., and Brett, M. (2007). Rhythm and beat perception in motor areas of the brain. J. Cogn. Neurosci. 19, 893-906. doi: 10.1162/jocn.2007.19.5.893

Grube, M., Lee, K. H., Griffiths, T. D., Barker, A. T., and Woodruff, P. W. (2010). Transcranial magnetic theta-burst stimulation of the human cerebellum distinguishes absolute, duration-based from relative, beat-based perception of subsecond time intervals. Front. Psychol. 1:171. doi: 10.3389/fpsyg.2010.00171

Hämäläinen, M., Hari, R., Ilmoniemi, R. J., Knuutila, J., and Lounasmaa, O. V. (1993). Magnetoencephalography: theory, instrumentation, and applications to noninvasive studies of the working human brain. Rev. Mod. Phys. 65, 413-497. doi: 10.1103/RevModPhys.65.413

Hannon, E. E., and Trehub, S. E. (2005a). Metrical categories in infancy and adulthood. Psychol. Sci. 16, 48-55. doi: 10.1111/j.0956-7976.2005.00779.x

Hannon, E. E., and Trehub, S. E. (2005b). Tuning in to musical rhythms: infants learn more readily than adults. Proc. Natl. Acad. Sci. U.S.A. 102, 12639-12643. doi: 10.1073/pnas.0504254102

Hari, R., and Salmelin, R. (1997). Human cortical oscillations: a neuromagnetic view through the skull. Trends Neurosci. 20, 44-49. doi: 10.1016/S01662236(96) 10065-5

Hommel, B., Müsseler, J., Aschersleben, G., and Prinz, W. (2001). The theory of event coding (TEC): a framework for perception and action planning. Behav. Brain Sci. 24, 849-937. doi: 10.1017/S0140525X01000103

Iversen, J. R., Repp, B. H., and Patel, A. D. (2009). Top-down control of rhythm perception modulates early auditory responses. Ann. N. Y. Acad. Sci. 1169, 58-73. doi: 10.1111/j.1749-6632.2009.04579.x

Ivry, R. B. (1996). The representation of temporal information in perception and motor control. Curr. Opin. Neurobiol. 6, 851-857. doi: 10.1016/S09594388(96)80037-7

Ivry, R. B., and Hazeltine, R. (1995). Perception and production of temporal intervals across a range of durations: evidence for a common timing mechanism. J. Exp. Psychol. Hum. Percept. Perform. 21, 3-18. doi: 10.1037/0096-1523.21.1.3

Jones, M. R., and Boltz, M. (1989). Dynamic attending and responses to time. Psychol. Rev. 96, 459-491. doi: 10.1037//0033-295X.96.3.459

Kello, C. T. (2003). Patterns of timing in the acquisition, perception, and production of speech. J. Phon. 31, 619-626. doi: 10.1016/S0095-4470(03)00017-2

Koch, G., Oliveri, M., and Caltagirone, C. (2009). Neural networks engaged in milliseconds and seconds time processing: evidence from transcranial magnetic 
stimulation and patients with cortical or subcortical dysfunction. Philos. Trans. R. Soc. Lond. B Biol. Sci. 364, 1907-1918. doi: 10.1098/rstb.2009.0018

Lakatos, P., Karmos, G., Mehta, A. D., Ulbert, I., and Schroeder, C. E. (2008). Entrainment of neuronal oscillations as a mechanism of attentional selection. Science 320, 110-113. doi: 10.1126/science.1154735

Large, E. W. (2008). "Resonating to musical rhythm: theory and experiment," in Psychology of Time, ed. S. Grondin (Cambridge: Emerald Group Publishing Limited), 189-231.

Large, E. W., and Jones, M. R. (1999). Dynamics of attending: how people track timevarying events. Psychol. Rev. 106, 119-159. doi: 10.1037/0033-295X.106.1.119

Manning, F., and Schutz, M. (2013). "Moving to the beat" improves timing perception. Psychon. Bull. Rev. 20, 1133-1139. doi: 10.3758/s13423-013-0439-7

McAuley, J. D., Jones, M. R., Holub, S., Johnston, H. M., and Miller, N. S. (2006). The time of our lives: life span development of timing and event tracking. J. Exp. Psychol. Gen. 135, 348-367. doi: 10.1037/0096-3445.135. 3.348

Näätänen, R., and Picton, T. (1987). The N1 wave response of the human electric and magnetic response to sound: a review and an analysis of the component structure. Psychophysiology 24, 375-425. doi: 10.1111/j.1469-8986.1987.tb00311.x

Nozaradan, S., Peretz, I., Missal, M., and Mouraux, A. (2011). Tagging the neuronal entrainment to beat and meter. J. Neurosci. 31, 10234-10240. doi: 10.1523/JNEUROSCI.0411-11.2011

Nozaradan, S., Peretz, I., and Mouraux, A. (2012). Selective neuronal entrainment to the beat and meter embedded in a musical rhythm. J. Neurosci. 32, 17572-17581. doi: 10.1523/JNEUROSCI.3203-12.2012

Patel, A., and Iversen, J. (2014). The evolutionary neuroscience of musical beat perception: the action simulation for auditory prediction (ASAP) hypothesis. Front. Syst. Neurosci. 13:57. doi: 10.3389/fnsys.2014.00057

Penhune, V. B., Zattore, R. J., and Evans, A. C. (1998). Cerebellar contributions to motor timing: a PET study of auditory and visual rhythm reproduction. J. Cogn. Neurosci. 10, 752-765. doi: 10.1162/0898929985 63149

Pfurtscheller, G., and Lopes da Silva, F. H. (1999). Event-related EEG/MEG synchronization and desynchronization: basic principles. Clin. Neurophysiol. 110, 1842-1857. doi: 10.1016/S1388-2457(99)00141-8

Pfurtscheller, G., Müller-Putz, G. R., Pfurtscheller, J., and Rupp, R. (2005). EEG-based asynchronous BCI controls functional electrical stimulation in a tetraplegic patient. EURASIP J. Appl. Signal Process. 2005, 3152-3155. doi: 10.1155/ASP.2005.3152

Phillips-Silver, J., and Trainor, L. J. (2005). Feeling the beat: movement influences infant rhythm perception. Science 308:1430. doi: 10.1126/science.1110922

Phillips-Silver, J., and Trainor, L. J. (2007). Hearing what the body feels: auditory encoding of rhythmic movement. Cognition 105, 533-546. doi: 10.1016/j.cognition.2006.11.006

Repp, B. H., London, J., and Keller, P. E. (2011). Perception-production relationships and phase correction in synchronization with two-interval rhythms. Psychol. Res. 75, 227-242. doi: 10.1007/s00426-010-0301-8

Repp, B. H., and Su, Y.-H. (2013). Sensorimotor synchronization: a review of recent research (2006-2012). Psychon. Bull. Rev. 20, 403-452. doi: 10.3758/s13423-0120371-2

Schubotz, R. I., Friederici, A. D., and von Cramon, D. Y. (2000). Time perception and motor timing: a common cortical and subcortical basis revealed by fMRI. Neuroimage 11, 1-12. doi: 10.1006/nimg. 1999.0514

Schubotz, R. I., and von Cramon, D. Y. (2001). Interval and ordinal properties of sequences are associated with distinct premotor areas. Cereb. Cortex 11, 210-222. doi: $10.1093 /$ cercor/11.3.210
Shahin, A. J., Roberts, L. E., Miller, L. M., McDonald, K. L., and Alain, C. (2007). Sensitivity of EEG and MEG to the N1 and P2 auditory evoked responses modulated by spectral complexity of sounds. Brain Topogr. 20, 55-61. doi: 10.1007/s10548-007-0031-4

Smith, N., Trainor, L., and Shore, D. (2006). The development of temporal resolution: between-channel gap detection in infants and adults. J. Speech Lang. Hear. Res. 49, 1104-1114. doi: 10.1044/1092-4388(2006/079)

Snyder, J. S., and Large, E. W. (2005). Gamma-band activity reflects the metric structure of rhythmic tone sequences. Brain Res. Cogn. Brain Res. 24, 117-126. doi: 10.1016/j.cogbrainres.2004.12.014

Su, Y. H., and Pöppel, E. (2012). Body movement enhances the extraction of temporal structures in auditory sequences. Psychol. Res. 76, 373-382. doi: 10.1007/s00426-011-0346-3

Teki, S., Grube, M., Kumar, S., and Griffiths, T. D. (2011). Distinct neural substrates of duration-based and beat-based auditory timing. J. Neurosci. 31, 3805-3812. doi: 10.1523/JNEUROSCI.5561-10.2011

Trainor, L. J. (2012). Musical experience, plasticity, and maturation: issues in measuring developmental change using EEG and MEG. Ann. N. Y. Acad. Sci. 1252, 25-36. doi: 10.1111/j.1749-6632.2012.06444.x

Trainor, L. J., Samuel, S. S., Desjardins, R. N., and Sonnadara, R. R. (2001). Measuring temporal resolution in infants using mismatch negativity. Neuroreport 12 , 2443-2448. doi: 10.1097/00001756-200108080-0003

Trehub, S. E., Schneider, B. A., and Henderson, J. L. (1995). Gap detection in infants, children, and adults. J. Acoust. Soc. Am. 98, 2532-2541. doi: 10.1121/1.414396

Trehub, S. E., and Thorpe, L. A. (1989). Infants' perception of rhythm: categorization of auditory sequences by temporal structure. Can. J. Psychol. 43, 217-229. doi: 10.1037/h0084223

Werner, L., Marean, G., Halpin, C., Spetner, N. B., and Gillenwater, J. (1992). Infant auditory temporal acuity: gap detection. Child Dev. 63, 260-272. doi: $10.2307 / 1131477$

Winkler, I., Háden, G. P., Ladinig, O., Sziller, I., and Honing, H. (2009). Newborn infants detect the beat in music. Proc. Natl. Acad. Sci. U.S.A. 106, 2468-2471. doi: 10.1073/pnas.0809035106

Zatorre, R. J., Chen, J. L., and Penhune, V. B. (2007). When the brain plays music: auditory-motor interactions in music perception and production. Nat. Rev. Neurosci. 8, 547-558. doi: 10.1038/nrn2152

Zentner, M., and Eerola, T. (2010). Rhythmic engagement with music in infancy. Proc. Natl. Acad. Sci. U.S.A. 107, 5768-5773. doi: 10.1073/pnas.1000121107

Conflict of Interest Statement: The authors declare that the research was conducted in the absence of any commercial or financial relationships that could be construed as a potential conflict of interest.

Received: 04 May 2014; accepted: 25 June 2014; published online: 11 July 2014. Citation: Cirelli LK, Bosnyak D, Manning FC, Spinelli C, Marie C, Fujioka T, Ghahremani A and Trainor LJ (2014) Beat-induced fluctuations in auditory cortical beta-band activity: using EEG to measure age-related changes. Front. Psychol. 5:742. doi: 10.3389/fpsyg.2014.00742

This article was submitted to Auditory Cognitive Neuroscience, a section of the journal Frontiers in Psychology.

Copyright (c) 2014 Cirelli, Bosnyak, Manning, Spinelli, Marie, Fujioka, Ghahremani and Trainor. This is an open-access article distributed under the terms of the Creative Commons Attribution License (CC BY). The use, distribution or reproduction in other forums is permitted, provided the original author(s) or licensor are credited and that the original publication in this journal is cited, in accordance with accepted academic practice. No use, distribution or reproduction is permitted which does not comply with these terms. 